\title{
TRIAGEM AUDITIVA NEONATAL COM EMISSÕES OTOACUSTICAS E REFLEXO COCLEO-PALPEBRAL: ESTUDO DA SENSIBILIDADE E ESPECIFICIDADE
}

\section{Newborn Hearing Screening with otoacoustic emissions and cochlear- palpebral reflex: sensitivity and specificity study}

\author{
Rosanna Mariangela Giaffredo Angrisani (1), Marcia Rumi Suzuki (2), Gustavo Ribeiro Pifaia (3), \\ Elaine Colombo Sousa ${ }^{(4)}$, Daniela Gil ${ }^{(5)}$, Marisa Frasson de Azevedo ${ }^{\left({ }^{(6)}\right.}$
}

\section{RESUMO}

Objetivo: analisar a especificidade e sensibilidade da TAN com emissões otoacústicas evocadas por estimulo transiente (EOAT) associadas à pesquisa do reflexo cócleo-palpebral (RCP), comparando-os aos resultados do Potencial Evocado Auditivo de Tronco Encefalico (PEATE). Método: a casuística do presente estudo foi composta por $369 \mathrm{RN}$ de risco para deficiência auditiva que foram submetidos à triagem com a captação das emissões otoacústicas evocadas por estímulo transiente e pesquisa do reflexo cócleo- palpebral. Os resultados foram comparados aos resultados do Potencial Evocado Auditivo de Tronco Encefalico (PEATE). Resultados: a incidência do tipo da perda auditiva na população geral foi de 4RN $(1,1 \%)$ com perda coclear, $22(5,9 \%)$ com perda condutiva, $2(0,5 \%)$ com espectro da neuropatia auditiva (ENA), 14 (3,8\%) com alteração central e $15(4,1 \%)$ com atraso de maturação da via auditiva. A TAN mostrou $100 \%$ de sensibilidade e $94,6 \%$ de especificidade na detecção de alterações cocleares e espectro da neuropatia auditiva; 77,3\% de sensibilidade e 94,6\% de especificidade na detecção de alterações condutivas e $42,9 \%$ de sensibilidade e $94,6 \%$ de especificidade na detecção de alterações centrais. Todos os achados evidenciaram diferenças estatisticamente significantes em relação aos neonatos auditivamente normais. Conclusão: este protocolo de TAN mostrou-se eficaz na detecção de RN com alterações de cocleares e espectro da neuropatia auditiva com sensibilidade e especificidade elevadas.

DESCRITORES: Recém-Nascido; Triagem Neonatal; Emissões Otoacústicas Espontâneas

(1) Fonoaudióloga clínica do Hospital São Paulo - HSP; Especialista em Audiologia Clínica pelo Conselho Federal de Fonoaudiologia, São Paulo,SP, Brasil.

(2) Fonoaudióloga clínica do Hospital São Paulo -HSP; Mestranda em Disturbios da Comunicação Humana pela Universidade Federal de São Paulo - UNIFESP, São Paulo,SP, Brasil.

(3) Fonoaudióloga clínica; Doutoranda do Programa de Pós Graduação em Fonoaudiologia da Universidade Federal de São Paulo - UNIFESP, São Paulo,SP, Brasil.

(4) Médico Otorrinolaringologista; Especialista em Otologia pela Universidade Federal de São Paulo - UNIFESP, São Paulo,SP, Brasil.

(5) Fonoaudióloga; Professora Adjunto do Departamento de FOnoaudiologia da UNIFESP; Doutora em Ciências pela Universidade Federal de São Paulo - UNIFESP, São Paulo, SP, Brasil.

\section{INTRODUÇÃO}

A deficiência auditiva vem recebendo mais atenção por parte dos órgãos públicos e privados da área da Saúde, devido à magnitude da alteração na qualidade de vida que provoca à criança deficiente auditiva e a seus familiares.

(6) Fonoaudióloga; Professor Associado da Escola Paulista de Medicina da Universidade Federal de São Paulo - UNIFESP; Doutorado em Distúrbios da Comunicação Humana pela Universidade Federal de São Paulo - UNIFESP, São Paulo,SP, Brasil.

Conflito de interesses: inexistente 
Nesse sentido, muito se tem investido na Triagem Auditiva Neonatal, reconhecidamente o melhor método de detecção desta deficiência que atinge 3:1000 neonatos sem risco e 4:1000 neonatos com risco e cujas causas genéticas somam cerca de $50 \%$ das perdas auditivas nesta população. ${ }^{1,2}$

A Triagem Auditiva Neonatal (TAN) compreende a realização de procedimentos comportamentais, eletroacústicos e/ou eletrofisiológicos para a identificação da deficiência auditiva. Seu objetivo é o de distinguir na população em geral, os indivíduos que apresentam um distúrbio auditivo daqueles que não o apresentam ${ }^{1}$.

O registro das Emissões Otoacústicas evocadas por estímulo transiente (EOAT) constitui um procedimento muito sensível na verificação da integridade coclear, uma vez que a resposta desaparece quando existe qualquer anomalia funcional significante nas células ciliadas da cóclea e/ou orelha média. Este método eletroacústico é objetivo, rápido e não invasivo ${ }^{1,3}$.

O registro do reflexo cócleo-palpebral (RCP) vem sendo bastante utilizado desde a década de 80 , por ser uma resposta comportamental que se apresenta em cerca de $90 \%$ a $100 \%$ das pessoas auditivamente normais. Desta forma, sua ausência deve ser investigada, pois pode ser decorrente de alteração da audição (Azevedo,1995, Lewis,1996, Diniz,2007) ${ }^{4-6}$. A ausência de RCP com presença de emissões otoacústicas pode ser indício de alteração retrococlear ${ }^{7}$.

Desta forma, o objetivo do presente estudo foi analisar a especificidade e sensibilidade da triagem auditiva neonatal com emissões otoacústicas evocadas por estimulo transiente associadas à pesquisa do reflexo cócleo-palpebral, comparandoos aos resultados do Potencial Evocado Auditivo de Tronco Encefálico (PEATE).

\section{MÉTODO}

Trata-se de um estudo observacional e transversal realizado em neonatos (RN) de risco para deficiência auditiva. Considerou-se RN de risco, todos os que permaneceram por mais de 5 dias em UTI neonatal, os RNs com histórico familiar de deficiência auditiva congênita e /ou consangüinidade; infecções congênitas tais como Sífilis, Toxoplasmose, Rubéola, Citomegalovirus, Herpes e infecção por HIV; anomalias crânio-faciais, incluindo de paviIhão auricular e meato acústico externo; hiperbilirrubinemia no nível de exsangüíneo transfusão; uso de ototóxicos; meningite bacteriana ou viral; asfixia neonatal ; ventilação mecânica por período maior que 5 dias; alcoolismo materno e/ou uso de drogas; convulsões neonatais; síndromes, ou desordens neurodegenerativas; traumatismo craniano; hemorragia ventricular; peso ao nascimento < a $1500 \mathrm{~g} \mathrm{e} /$ ou PIG (pequeno para a idade gestacional);otites médias recorrentes / persistentes ${ }^{2,7}$.

A casuística do presente estudo foi composta por $369 \mathrm{RN}$ de risco para deficiência auditiva, sendo183 RN internados em UTI Neonatal e 186 de Alojamento Conjunto, na maternidade do Hospital São Paulo vinculado à UNIFESP, no período de maio de 2009 a setembro de 2010. Os neonatos foram selecionados após a leitura do prontuário do RN e anamnese com a mãe, identificando-se assim, os $\mathrm{RN}$ de risco.

Para a realização da TAN, a criança permaneceu no berço ou no colo da mãe, em sono natural.

Todos os RN foram submetidos à TAN com a captação das emissões otoacústicas evocadas por estímulo transiente (EOAT) e pesquisa do reflexo cócleo- palpebral (RCP). O PEATE clínico foi realizado em todos os neonatos na mesma semana.

Antes do PEATE, todos os neonatos passaram por avaliação com médico otologista, que realizou a otoscopia, por meio de otoscópio marca Welch Allyn para visualização da membrana timpânica.

A pesquisa do Reflexo Cócleo-palpebral (RCP) foi feita mediante a percussão forte da campânula grande do instrumento musical Agogô (100 dB NPS), de acordo com os parametros de avaliação de Azevedo (1995) ${ }^{4}$, a 20 centímetros da orelha do RN.

A captação das EOAT foi realizada no equipamento automático portátil AccuscreenPRO, marca GN Otometrics. Para iniciar o teste, a sonda auricular foi posicionada no meato acústico externo do neonato para a emissão do estímulo acústico.

Para a realização do PEATE, a limpeza da pele foi realizada em Fz e nas mastóides direita e esquerda $\left(M_{2}\right.$ e $\left.M_{1}\right)$, para fixação dos eletrodos descartáveis (Meditrace 200 - marca Kendal), obedecendo à norma IES 10-20 (International Electrode System). O exame foi realizado com o equipamento modelo Smart- EP, marca Intelligent Hearing Systems. Na sequência, o fone de inserção (3-A) foi posicionado no meato acústico externo do RN para a emissão do estímulo acústico. O estímulo acústico utilizado foi o clique de polaridade rarefeita, numa velocidade de apresentação de $27,7 / \mathrm{seg}$, duração de 0,1 milissegundos (ms), janela de gravação de $12 \mathrm{~ms}$, sendo empregado um total de 2048 estímulos apresentados em duas séries em cada intensidade.

Foram aplicados dois protocolos de pesquisa: o da integridade da via auditiva (80 dB NAn), com aplicação da tabela de valores das latências absolutas e interpicos das ondas I, III e V de acordo com a idade gestacional; e o da estimativa do nível de 
audição (presença da onda $V$ a 30 dB NAn, como valor de corte para audição normal para cliques), seguindo o critério de normalidade do equipamento Smart-EP Intelligent Hearing (Jacobson, 1985)8.

Para a análise das respostas do PEATE, foram medidas as latências absolutas das ondas I, III, V e interpicos I-III, III-V, I-V a $80 \mathrm{~dB}$ nNA e presença da onda $V$ a $30 \mathrm{~dB}$ NAn.

Os critérios de analise das respostas auditivas permitiram identificar:

- RNs com audição normal: presença de EOAT e PEATE com protocolo neurológico normal e presença da onda $\mathrm{V}$ a $30 \mathrm{dBNAn}$, como valor de corte para audição normal para cliques.

- RNs com perda coclear: ausência de EOAT, e PEATE com protocolo neurológico normal e presença da onda $\mathrm{V}$ em níveis superiores a 30 dB NAn.

- RNs com perda condutiva: ausência de EOAT com avaliação otorrinolaringológica alterada. PEATE com protocolo neurológico alterado (padrão condutivo - latências absolutas aumentadas das ondas I, III e V com interpicos preservados- Hall, 2006) e presença da onda $V$ em níveis superiores a $30 \mathrm{~dB}$ NAn.

- RNs com alteração retrococlear: presença de EOAT e PEATE com protocolo neurológico alterado (aumento das latências absolutas das ondas I, III e V e/ou interpicos I-/III, III-V e I-V) com presença da onda $\mathrm{V}$ a $30 \mathrm{dBNAn}$, como valor de corte para audição normal para cliques .

- RNs com atraso maturacional: presença de EOAT e PEATE com protocolo neurológico alterado (aumento da latência absoluta da onda III e/ou V) no primeiro PEATE, com normalização das mesmas no intervalo de 30 dias.

- RNs com Espectro da Neuropatia Auditiva: presença de EOAT e PEATE com protocolo neurológico alterado (ausência de respostas) com presença de microfonismo coclear.

O projeto do estudo foi aprovado pelo Comitê de Ética e Pesquisa da Universidade Federal de São Paulo sob o ํo CEP 1362/08. Os responsáveis foram informados sobre os procedimentos do estudo e assinaram o termo de consentimento livre e esclarecido a fim de assegurar os preceitos éticos na realização de pesquisa com seres humanos.

A análise estatística foi realizada por um profissional da área, empregando-se o teste de Qui-Quadrado para medir o grau de relação e/ou associação entre EOAT+RCP e PEATE. Foram calculadas: Acurácia, Sensibilidade, Especificidade, Valor Preditivo Positivo e Negativo (figura 1).

\begin{tabular}{|c|c|c|c|}
\hline & Doente & Não Doente & Total \\
\hline Positivo & $\mathrm{A}$ & $\mathrm{B}$ & $\mathrm{A}+\mathrm{B}$ \\
\hline Negativo & $\mathrm{C}$ & $\mathrm{D}$ & $\mathrm{C}+\mathrm{D}$ \\
\hline Total & $\mathrm{A}+\mathrm{C}$ & $\mathrm{B}+\mathrm{D}$ & $\mathrm{A}+\mathrm{B}+\mathrm{C}+\mathrm{D}$ \\
\hline
\end{tabular}

Figura 1 - Cálculo da Sensibilidade e Especificidade

Sensibilidade $=$ porcentagem de doentes designados como positivos com o teste de triagem $A /(A+C)$.

Especificidade $=$ porcentagem de não-doentes designados como negativos com o teste de triagem $D /(B+D)$.

Falso-positivo $=\mathrm{B}$; Falso-negativo $=\mathrm{C}$

Valor Preditivo Positivo $=A /(A+B)$

Valor Preditivo Negativo $=D /(C+D)$

Acurácia $=(A+D) /(A+B+C+D)$

O nível de significância definido para o trabalho foi de $0,05(5 \%)$. Os intervalos de confiança construídos ao longo do trabalho foram construídos com 95\% de confiança estatística.

\section{RESULTADOS}

A incidência de alterações auditivas nos $369 \mathrm{RN}$ de UTI foi de $15,4 \%$ (57 neonatos), sendo que do total, $312 \mathrm{RNs}(84,6 \%)$ foram diagnosticados como auditivamente normais. (figura 2 )

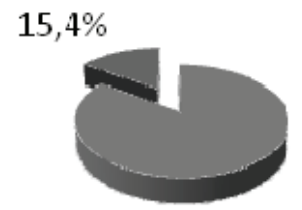

$84,6 \%$

- Normal =Alterado

Figura 2 - Incidência de alterações auditivas em recém nascidos de alto risco 
A análise do tipo de alteração para os 57 sujeitos com alteração auditiva mostrou que a alteração mais prevalente foi a do tipo condutiva $(38,6 \%)$, $7,0 \%$ apresentou alteração do tipo coclear, $24,6 \%$ mostrou alteração do tipo central, 3,5\% com espectro da neuropatia auditiva. Em 15 RNs houve atraso de maturação com recuperação no segundo exame (figura 3).

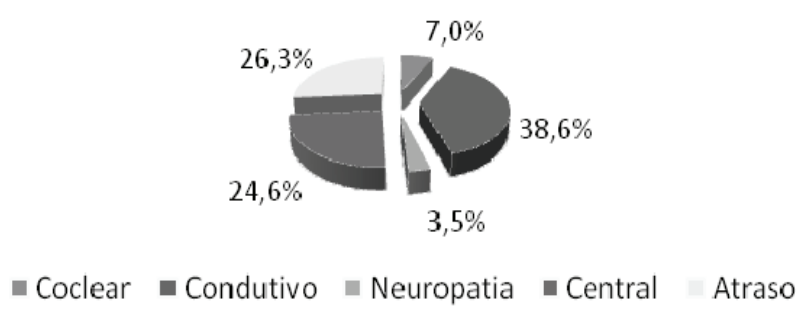

Figura 3 - Distribuição do Tipo de Alteração

Portanto, a incidência na população geral foi de 4RN $(1,1 \%)$ com perda coclear, $22(5,9 \%)$ com perda condutiva, $2(0,5 \%)$ com espectro da neuropatia auditiva (ENA), $14(3,8 \%)$ com alteração central e $15(4,1 \%)$ com atraso de maturação (figura 4).

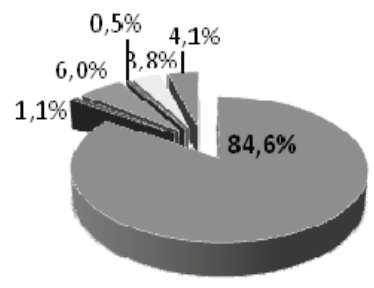

$$
\begin{aligned}
& \text { normal }=\text { Coclear }=\text { Condutivo } \\
& \text { neuropatia Central }=\text { Atraso }
\end{aligned}
$$

Figura 4 - Distribuição das incidências dos tipos de alteração

Os resultados da triagem auditiva neonatal em relação à identificação dos casos de auditivas cocleares encontram-se descritos na tabela 1.

$\mathrm{Na}$ comparação dos resultados da TAN com $\mathrm{EOAT+RCP}$ entre RN auditivamente normais e RN com alterações auditivas cocleares, observou-se diferença estatisticamente significante $(p<0,001)$, sendo que $100 \%$ dos RNs com perdas auditivas de origem coclear foram detectados pela TAN (tabela 1).

A análise dos resultados mostrou também sensibilidade de $100 \%$, especificidade de $94,6 \%$, com valores preditivos positivo de $10,5 \%$ e negativo de 100\%. A acurácia da TAN foi de $94,6 \%$.

Os resultados da triagem auditiva neonatal em relação à identificação dos casos de espectro da neuropatia auditiva encontram-se descritos na tabela 2.

A TAN mostrou sensibilidade de $100 \%$, isto é, detectou todos casos de espectro da neuropatia auditiva (ENA), de acordo com a analise comparativa, onde foram encontradas diferenças estatisticamente significantes entre os RN auditivamente normais e os RN com ENA (tabela 2). A análise mostrou ainda, especificidade da TAN de 94,6\%, com valores preditivos positivo de $10,5 \%$ e negativo de $100 \%$. A acurácia da TAN foi de $94,6 \%$.

Os resultados da triagem auditiva neonatal em relação à identificação das alterações centrais encontram-se descritos na tabela 3.

A análise comparativa dos resultados mostra que a TAN com EOAT + RCP identificou apenas $42,9 \%$ (sensibilidade) dos RN com alterações de origem central, com especificidade de $94,6 \%$.Esses dados mostram também que este protocolo de TAN teve acurácia de $92,4 \%$ com valores preditivos positivo de $26,1 \%$ e negativo de $97,6 \%$, ou seja, $97,6 \%$ que passam na TAN tem a probabilidade de serem audiologicamente normais.

Os resultados da triagem auditiva neonatal em relação à identificação das alterações condutivas encontram-se descritos na tabela 4.

$\mathrm{Na}$ comparação dos resultados da triagem com EOAT+RCP entre RN auditivamente normais e RN com alterações auditivas condutivas, observou-se diferença estatisticamente significante $(p<0,001)$, sendo que o exame mostrou boa sensibilidade, uma vez que $77,3 \%$ dos RNs com perdas auditivas condutivas foram detectados pela TAN, evidenciando especificidade de $94,6 \%$. Os resultados estatísticos evidenciaram acurácia de 93,5\%, valores preditivos positivo de $50 \%$ e negativo de $98,3 \%$ (tabela 4 ).

\section{DISCUSSÃO}

A ocorrência de perda coclear observada no presente estudo $(1,1 \%)$ foi superior à encontrada em estudo que utilizou protocolo similar de TAN (EOAT + RCP), encontrando $7 \mathrm{RNs}, 0,2 \%$ do total, com perda auditiva neurossensorial bilateral ${ }^{9}$. Porém, a ocorrencia de perda coclear no presente estudo foi inferior à obtida na literatura para recém nascidos de UTI neonatal (3\% a 5\%) ${ }^{10}$. Suppiej et al (2007) observaram $2,9 \%$ de perda auditiva congênita em RN de risco ${ }^{11}$. De fato, desde 1999, a Academia Americana de Pediatria já relatara incidência de 
Tabela 1 - Achados nas EOAT+ RCP nas alterações auditivas cocleares

\begin{tabular}{ccccccc}
\hline & \multicolumn{2}{c}{ Normal } & \multicolumn{2}{c}{ Coclear } & \multicolumn{2}{c}{ Total } \\
\cline { 2 - 7 } & $\mathbf{N}$ & $\%$ & $\mathbf{N}$ & $\%$ & $\mathbf{N}$ & $\%$ \\
\hline Passou & 298 & $94,6 \%$ & 0 & $0,0 \%$ & 298 & $93,4 \%$ \\
Falhou & 17 & $5,4 \%$ & 4 & $100 \%$ & 21 & $6,6 \%$ \\
Total & 315 & $98,7 \%$ & 4 & $1,3 \%$ & 319 & $100 \%$ \\
P-valor & $<0,001$ & & & & & \\
\hline
\end{tabular}

Acuracia 94,6\% - Sensibilidade 100\% - Especificidade 94,6\% - VP+10,5\% - VP - 100\%

Tabela 2 - Achados nas EOAT+ RCP no espectro da neuropatia auditiva

\begin{tabular}{ccccccc}
\hline & \multicolumn{2}{c}{ Normal } & \multicolumn{2}{c}{ Neuropatia } & \multicolumn{2}{c}{ Total } \\
\cline { 2 - 7 } & $\mathbf{N}$ & $\%$ & $\mathbf{N}$ & $\%$ & $\mathbf{N}$ & $\%$ \\
\hline Passou & 298 & $94,6 \%$ & 0 & $0,0 \%$ & 298 & $94,0 \%$ \\
Falhou & 17 & $5,4 \%$ & 2 & $100 \%$ & 19 & $6,0 \%$ \\
Total & 315 & $99,4 \%$ & 2 & $0,6 \%$ & 317 & $100 \%$ \\
P-valor & $<0,001$ & & & & & \\
\hline Acurat & & & &
\end{tabular}

Acuracia 94,6\% - Sensibilidade 100\% - Especificidade 94,6\% - VP+10,5\% - VP - 100\%

Tabela 3 - Achados nas EOAT+ RCP nas alterações auditivas centrais

\begin{tabular}{ccccccc}
\hline & \multicolumn{2}{c}{ Normal } & \multicolumn{2}{c}{ Central } & \multicolumn{2}{c}{ Total } \\
\cline { 2 - 7 } & $\mathbf{N}$ & $\%$ & $\mathbf{N}$ & $\%$ & $\mathbf{N}$ & $\%$ \\
\hline Passou & 298 & $94,6 \%$ & 8 & $57,1 \%$ & 306 & $93,0 \%$ \\
Falhou & 17 & $5,4 \%$ & 6 & $42,9 \%$ & 23 & $7,0 \%$ \\
Total & 315 & $95,7 \%$ & 14 & $4,3 \%$ & 329 & $100 \%$ \\
P-valor & $<0,001$ & & & & & \\
\hline
\end{tabular}

Acuracia 92,4\% - Sensibilidade 42,9\% - Especificidade 94,6\% - VP+ 26,1\% - VP - 97,6\%

Tabela 4 - Achados nas EOAT+ RCP nas alterações auditivas condutivas

\begin{tabular}{ccccccc}
\hline & \multicolumn{2}{c}{ Normal } & \multicolumn{2}{c}{ Condutivo } & \multicolumn{2}{c}{ Total } \\
\cline { 2 - 7 } & $\mathbf{N}$ & $\%$ & $\mathbf{N}$ & $\%$ & $\mathbf{N}$ & $\%$ \\
\hline Passou & 298 & $94,6 \%$ & 5 & $22,7 \%$ & 303 & $89,9 \%$ \\
Falhou & 17 & $5,4 \%$ & 17 & $77,3 \%$ & 34 & $10,1 \%$ \\
Total & 315 & $93,5 \%$ & 22 & $6,5 \%$ & 337 & $100 \%$ \\
P-valor & $<0,001$ & & & & & \\
\hline
\end{tabular}

Acuracia 93,5\% - Sensibilidade 77,3\% - Especificidade 94,6\% - VP+50\% - VP - 98,3\%

$2 \%$ a $4 \%$ de perda auditiva em neonatos de risco ${ }^{12}$. Outros autores também verificaram ocorrência de perda neurossensorial entre $2,2 \%$ e $3,2 \%$ na população de UTI neonatal ${ }^{13-15}$, Todavia, outro estudo encontrou $0,32 \%$ de $\mathrm{RN}$ com perda coclear, do total da população estudada ${ }^{16}$. Tais diferenças podem ser justificadas pelo tipo de população avaliada que incluiu bebes com e sem risco.

No presente estudo, todos os RN com perda auditiva confirmada tiveram ausência de RCP, o que concorda com o estudo de Barreira-Nielsen, que obteve os mesmos resultados. 
O estudo da associação entre a triagem auditiva realizada com EOAT associada à pesquisa do RCP e os resultados do PEATE clínico, demonstrou relação estatisticamente significante com valores elevados de acurácia (94,7\%) de sensibilidade $(100 \%)$ e especificidade $(94,6 \%)$.

$\mathrm{O}$ valor preditivo negativo foi bastante elevado $(100 \%)$, ou seja, quem passa na TAN é auditivamente normal. Entretanto, valor preditivo positivo foi baixo $(10,5 \%)$, ou seja, se a criança falhar na TAN terá apenas $10,5 \%$ de chance de apresentar perda auditiva coclear. Este fato se deve ao elevado número de falsos positivos $(5,4 \%)$. Tais resultados coincidem com estudo cujos falsos positivos foram de $6,3 \%{ }^{15}$.

A elevada ocorrência de falsos positivos com o protocolo de EOAT associado ao RCP poderia explicar a recomendação do uso do PEATE em neonatos de risco por gerar menor índice de encaminhamentos para diagnóstico ${ }^{17}$. Entretanto, a possibilidade de não identificar perdas cocleares ascendentes pelo PEATE, uma vez que os limiares eletrofisiológicos obtidos com este procedimento coincidem com os limiares audiologicos entre $2 \mathrm{KHz}$ e $4 \mathrm{KHz}$ ou entre $3 \mathrm{KHz}$ a $6 \mathrm{KHz}$, dependendo do equipamento utilizado ${ }^{17}$, poderia se confrontar com a vantagem da reduzida taxa de encaminhamentos. É por isso, que o uso do PEATE com tone burst no diagnóstico de neonatos vem sendo recomendado na literatura para caracterizar a configuração do audiograma ${ }^{18}$.

Em estudo anterior, Angrisani et al (no prelo) obtiveram valores baixos de especificidade (75\% para a orelha direita e $60 \%$ para a orelha esquerda) com o uso do PEATE automático isolado como triagem ${ }^{19}$.

A triagem auditiva realizada com EOAT associada ao RCP foi bastante sensível na identificação dos dois casos de espectro da neuropatia auditiva (ENA). Vale ressaltar que a falha ocorreu pela ausência de RCP com presença de EOAT. Houve relação estatisticamente significante entre os resultados com elevada sensibilidade(100\%) e especificidade $(94,6 \%)$. A acurácia foi de $94,6 \%$. Portanto, o uso do RCP associado ao uso das EOAT auxiliou na identificação do ENA em crianças de alto risco com valores elevados de sensibilidade e especificidade. Porém, a elevada ocorrência de falsos positivos $(5,4 \%)$ elevaria o custo do programa em saúde pública.

A baixa ocorrência de ENA (0,5\%) observada no presente estudo, já era esperada, estando de acordo com dados na literatura, que tem relatado prevalência de ENA em população de alto risco entre $0,2 \%$ a $4 \%{ }^{20-22}$. A ocorrência do ENA na popu- lação geral tem sido descrita como bem inferior, entre $0,01 \%$ a $0,04 \%^{13,22}$.

Alguns estudos relatam que $4 \%$ a $8 \%$ das crianças com perda auditiva de grau profundo apresentam ENA ${ }^{23}$. A prevalência de ENA também aumenta em neonatos de risco, em especial nos casos de hiperbilirrubinemia ${ }^{19,22}$.

A maior ocorrência do ENA em neonatos de risco, levou o $\mathrm{JCIH}$ a recomendar a inclusão do PEATE na triagem de RN que permaneceram por mais de cinco dias em UTI ${ }^{2}$.

A ocorrência de RN com alterações centrais observada no presente estudo $(4,3 \%)$ foi similar à obtida em estudo que obteve incidencia de $4,28 \%{ }^{14}$.

O estudo comparativo dos resultados da TAN com EOAT associado ao RCP nos RN normais e os com alterações centrais mostrou relação estatisticamente significante, porém com baixa sensibilidade para identificação das alterações centrais $(42,9 \%)$, Em trabalho anterior, Angrisani et al(no prelo) verificaram que o uso do PEATEa identificou mais de $99,4 \%$ das alterações centrais com valor preditivo positivo de $97 \%$, ou seja, se o neonato falhasse no PEATEa teria $97 \%$ de chance de apresentar alteração central. Entretanto, os valores de especificidade com o PEATEa foram medianos $(54,5 \%-63,6 \%)$, inferiores ao $94,6 \%$ obtido no presente estudo, utilizando o RCP. Tais resultados reforçam a importância da inclusão do PEATEa na TAN de RN de UTI, complementando a avaliação por EOAT mas não invalida a importância da avaliação comportamental com eliciação do RCP, pois a avaliação comportamental é considerada parte importante da investigação ao possibilitar informações de como os recursos auditivos são utilizados pela criança e por avaliar as vias centrais da audição. A presença de reações comportamentais sugere integridade das vias auditivas e ausência de perda auditiva severa ${ }^{25}$.

No presente estudo o valor preditivo negativo para alterações centrais foi elevado $(97,4 \%)$, ou seja, o neonato que passou na triagem tem $97,4 \%$ de chance de ser audiologicamente normal. $O$ valor preditivo positivo mostrou-se baixo, ou seja, se a criança falhou na triagem tem apenas $26,1 \%$ de chance de ser auditivamente alterada devido ao elevado número de falsos positivos.

Em relação ao comprometimento condutivo, houve relação significante entre os resultados, com acurácia de $93,5 \%$, com alta especificidade $(94,6 \%)$ e sensibilidade regular $(77,3 \%)$. A especificidade encontrada foi bem superior à obtida em estudo anterior dos mesmos autores, realizado com PEATEa (de $33,3 \%$ e $42,9 \%$ para as orelhas direita e esquerda respectivamente) ${ }^{18}$. O fato de deixar passar na TAN as perdas condutivas gera menor 
índice de encaminhamentos e por esta razão o uso do PEATEa tem sido considerado como vantajoso ${ }^{26}$. Entretanto, estudo prospectivo realizado, concluiu que o neonato que falha na TAN por alteração de orelha média tem mais chance de apresentar otites de repetição no primeiro ano de vida ${ }^{27}$. Outro estudo concluiu que o RN que falha por alteração de orelha média tem 2,9 vezes mais chance de ter alterações condutivas no primeiro ano de vida ${ }^{14}$. O curto período de internação hospitalar após o nascimento pode elevar a taxa de exames falsopositivos. A presença de líquido amniótico e vérnix caseoso no conduto auditivo podem comprometer a transmissão dos sons e levar à falha nas emissões. Alguns autores sugerem que a repetição seriada dos testes que acusam alteração, ainda durante a internação, possa minimizar este problema. A repetição do exame exigiria um período maior de permanência do RN no hospital28-30.

Nos programas de TAN, recomenda-se um índice de respostas falso positivo inferior a $4 \%{ }^{31}$. As respostas falso positivo obtidas com o protocolo estudado foram superiores ao recomendado $(5,4 \%)$.

EmestudoanteriorcomPEATEaosfalso positivos foram inferiores a $0,6 \%$. Valores de falso-positivos, inferiores a $3 \%$, tem sido recomendados visto que, em altas taxas, além de gerar ansiedades familiares desnecessárias, aumentam o número de retornos, elevando o custo do programa de $\mathrm{TAN}^{12,32}$.

No presente estudo não foram encontrados falsos negativos na identificação de perdas cocleares e espectro da neuropatia auditiva.

\section{CONCLUSÃO}

O protocolo com emissões otoacusticas associado ao reflexo cocleo-palpebral mostrou-se eficaz para a detecção de RN com alterações cocleares e espectro da neuropatia auditiva.

\section{AGRADECIMENTOS}

À Fundação de Amparo à Pesquisa do Estado de São Paulo - FAPESP pelo financiamento deste estudo, sem o qual não haveria a possibilidade de realização.

Ao Hospital São Paulo por ceder espaço físico adequado à realização dos procedimentos.

\section{ABSTRACT \\ Purpose: to study the specificity and sensitivity of NHS with otoacoustic emissions transient stimulus (TEOAE) associated with cochlear-palpebral reflex (CPR), and comparing the results of Auditory Evoked Potential (BAEP). Method: three hundred and sixty-nine neonates under risk of hearing loss were evaluated. Evaluation procedures consisted of transient otocoustic emissions(TOAEs), cochlear-palpebral reflex (CPR), and ABR diagnostic carried out in the same week. Results: the incidence in the general population was 4 neonates (1.1\%) with cochlear hearing loss, $22(5.9 \%)$ with conductive hearing loss, $2(0.5 \%)$ with auditory neuropathy, $14(3.8 \%)$ with central abnormalities and $15(4.1 \%)$ with delayed maturation of the auditory pathway. The TAN showed $100 \%$ sensitivity and $94.6 \%$ specificity in detecting the spectrum of cochlear and auditory neuropathy, $77.3 \%$ sensitivity and $94.6 \%$ specificity in detecting conductive and $42.9 \%$ and $94.6 \%$ specificity while detecting central abnormalities. All findings showed statistically significant differences when compared to normal auditory neonates. Conclusion: this protocol was effective to detect in neonates with cochlear hearing loss and auditory neuropathy with high sensitivity and specificity.}

KEYWORDS: Infant, Newborn; Neonatal Screening; Otoacoustic Emissions, Spontaneous 


\section{REFERÊNCIAS}

1. Lewis DR, Marone SAM, Mendes BCA, Cruz OLM, Nóbrega M. Comitê multiprofissional em saúde auditiva - COMUSA Braz J Otorhinolaryngol. 2010;76 (1):121-8.

2. Joint Committee on Infant Hearing, Year 2007 Position Statement: Principles and Guidelines for Early Hearing Detection and Intervention Programs. J Pediatr.. 2007; 120(4): 898-921.

3. Azevedo M.F. Programa de Triagem Auditiva e Acompanhamento Audiológico realizado na UNIFESP. In:.Figueiredo MS. Emissões Otoacústicas e BERA São Paulo, Coleção CEFAC, 2003; cap.2, p.73.

4. Azevedo M.F, Vieira RM, Vilanova LC. Desenvolvimento Auditivo de Crianças Normais e de Alto Risco.1 ${ }^{a}$ Ed.São Paulo: Plexus, 1995.

5. Lewis DR. As habilidades auditivas do recémnascido e a triagem auditiva

neonatal. In: Andrade CRF. Fonoaudiologia em berçário normal e de risco. São Paulo:Lovise,1996. p.149-68.

6. Diniz M.G. Pesquisa da habituação do Reflexo Cócleo-Palpebral e do Reflexo De Sobressalto em lactentes de baixo risco para a deficiência auditivaTrabalho de conclusão de curso de graduação apresentado da Faculdade de Fonoaudiologia na Universidade Federal de Minas Gerais, 2007.

7. Azevedo MF.Triagem Auditiva Neonatal. In: Tratado de Fonoaudiologia/ [org] Fernandes FDM;Mendes BCA; Navas ALGP. $2^{2}$ ed.-São Paulo: Roca, 2009-pg.65-77.

8. Jacobson, John T. The Auditory Brainstem Response. Copyright 1985 by Allyn and Bacon. Reprinted by permission. All ages are referenced from conception

9. Mattos, W. M. de et al. Análise da implantação de programa de triagem auditiva neonatal em um hospital universitário. Rev. Bras. Otorrinolaringol. [online],2009;75(2): 237-44. ISSN 0034-7299.

10. Hilú MRPB, Zeigelboim BS. O conhecimento,a valorização da triagem auditiva neonatal e a intervenção precoce da perda auditiva. Rev CEFAC.2007; 9(4):563-70.

11. Suppiej A, Rizzardi E, Zanardo V, Franzoi M, Ermani M, Orzan E. Reliability of hearing screening in high-risk neonates:comparative study of otoacoustic emission, automated and conventional auditory brainstem response. Clinical Neurophysiology, 2007; 118(4), 869-76.

12. American Academy of Pediatrics. Task force on newborn and infant hearing. Pediatrics, 1999;103(2):527-9.

13. White KR, Vohr BR, Meyer S, Widen, JE, Johnson JL, Gravel, JS et al. A multisite study to examine the efficacy of the otoacoustic emission/ automated auditory brainstem response newborn hearing screening protocol: Research design and results of the study. A.m J. of Audiol. 2005;14, S186-S99.

14. Pereira PKS, Martins AS, Vieira MR, Azevedo MFde. Programa de triagem auditiva neonatal: associação entre perda auditiva e fatores de risco. Pró-Fono Revista de Atualização Científica, Barueri (SP), 2007;19(3):267-78.

15. Lima MCMP, Rossi TRF, Françozo MFC, Marba ST, Lima GML, Santos MFC. Detecção de perdas auditivas em neonatos de um hospital público Rev Soc Bras Fonoaudiol. 2010; 15(1):1-6.

16. Barreira-Nielsen C, Futuro Neto HA, Gattaz G. Processo de implantação de Programa de Saúde Auditiva em duas maternidades públicas. Rev Soc Bras Fonoaudiol. 2007; 12(2):99-105.

17. Matas CG; Neves IF. Potenciais Evocados Auditivos de Curta Latência. In: Tratado de Fonoaudiologia/[org]Fernandes FDM;Mendes BCA; Navas ALGP. 2 ${ }^{\mathrm{a}}$ Ed.-São Paulo: Roca, 2009-p.85-98.

18. Ribeiro FM; Carvallo RMM. Tone evoked ABR in normal hearing neonates. Int J Audiol.2008;47:21-9. 19. Angrisani RMG, Suzuki MR; Pifaia GR, Testa JR, Sousa EC, Gil D, Azevedo MF. Peate automático em recém nascidos de risco: estudo da sensibilidade e especificidade.Rev.CEFAC ( no prelo),2010.

20. Ngo,RYS Tan HKK Balakrishnan $A$ et al Auditory neuropathy/auditory dys-sincrony detected by universal newborn screening. Int $J$ Pediatric Otorhrinolayngol 2006; 70:1299-306.

21. Stein L K, Tremblay K, Pasternak J, Banerjee S, Lindemann K, Kraus N. Brainstem abnormalities in neonates with normal otoacoustic emissions. Semin Hear 1996; 17:197-213.

22. Berlin C, Hood L, Rose K. On renaming auditory neuropathy as auditory dys-synchrony. Audiol Today.2001; 13:15-7.

23. Kirkim,G; Serbetcioglu,B; Erdag TK et al. The frequency of neuropathy detected by universal newborn hearing screening program. Int J Pediatric Otorhinolaryngol 2008;72:1461-69.

24. Foerst $A$; Beutner,D;Lang-Roth $R$ et al Prevalence of auditory neuropathy/ synaptopathy in a population of children with profound hearing loss. Int J Pediatric Otorhrinolayngol 2006;70: 1415-22.

25. Marone MR, Lichtig I, Marone SAM. Recémnascidos gerados por mães com alto risco gestacional: estudo das emissões otoacústicas produtos de distorção e do comportamento auditivo. Rev Bras Otorrinolaringol. 2002;68(2):230-7.

26. Freitas VS, Alvarenga KF, Bevilacqua MC, Martinez MAN, Costa OA. Critical analysis of three newborn hearing screening protocols (original title: Análise crítica de três protocolos de triagem 
auditiva neonatal). Pró-Fono Revista de Atualização Científica. 2009; 21(3):201-6.

27. Doyle KJ; Kong, YY; Strobel K.; Dallaire,P; Ray, RM. Neonatal middle ear effusion predicts chronic otitis media with effusion. Otol. Neurotol. 2004; 25(3): 318-22.

28. Wroblewska-Seniuk K, Chojnacka K, Pucher B, Szczapa J, Gadzinowski J, Grzegorowski M. The results of newborn hearing screeningby means of transient evoked otoacoustic emissions. Int J Pediatr Otorhinolaryngol. 2005; 69 (10):1351-7.

29. Korres SG, Balatsouras DG, Nikolopoulos T, Korres GS, Ferekidis E. Making Universal Newborn Hearing Screening a success. Int $\mathrm{J}$ Pediatr Otorhinolaryngol. 2006;70(2):241-6.
30. Korres SG, Balatsouras DG, Gkoritsa E, Eliopoulos P, Rallis E, Ferekidis E.Success rate on newborn and follow up screening of hearing using otoacoustic emissions. Int $\mathrm{J}$ Pediatr Otorhinolaryngol. 2006; 70(6):1039-43.

31. NIH National Institute of Health Consensus Development Conference Statement. Early identification of hearing impairment in infants and young children. Int J Pediatr Otorhinolaryngol, 1993; 27:215-27.

32. Porter HL, Neely ST, Gorga MP.Using benefitcost ratio to select Universal Newborn Screening test criteria. Ear Hear. 2009; 30(4): 447-57.

http://dx.doi.org/10.1590/S1516-18462011005000108

RECEBIDO EM: 30/12/2010

ACEITO EM: 30/04/2011

Endereço para correspondência:

Rosanna Giaffredo Angrisani

Rua Martiniano de Carvalho, 548-41 - Paraiso

São Paulo - SP

CEP: 01321-000

E-mail: roangrisani@gmail.com 